\title{
Tratamentos na sanidade e germinação de Lonchocarpus sericeus (Poir.) Kunth ex DC
}

\author{
Lenita Gonçalves da Costa ${ }^{1 *}$, Ediglécia Pereira de Almeida ${ }^{*}$, Pedro Tôrres Ferreira Moreira \\ Montenegro ${ }^{1}$, Gilvan José Campelo dos Santos ${ }^{1}$.
}

\begin{abstract}
RESUMO: Essa pesquisa teve como objetivo verificar os efeitos do extrato de Gingiber officinale na germinação e sanidade de sementes de Lonchocarpus sericeus e incentivar o uso de alternativas naturais na obtenção de controle de microorganismos associados às sementes. Para a realização do teste de germinação as sementes foram submetidas ao processo de superação de dormência tegumentar através de escarificação mecânica com lixa número 80, e posteriormente submetidas aos tratamentos com extrato de gengibre nas seguintes concentrações $\mathrm{T} 1(\mathrm{Controle})=40 \mathrm{ml}$ de $\mathrm{H}_{2} \mathrm{O}$ estéril, T2= $10 \mathrm{ml}$ de extrato $+30 \mathrm{ml}$ de $\mathrm{H}_{2} \mathrm{O}$ estéril, T3= $20 \mathrm{ml}$ de extrato $+20 \mathrm{ml} \mathrm{de} \mathrm{H}_{2} \mathrm{O}$ estéril, T4= $30 \mathrm{ml}$ de extrato $+10 \mathrm{ml}$ de $\mathrm{H}_{2} \mathrm{O}$ estéril e T5= $40 \mathrm{ml}$ de extrato. $\mathrm{O}$ teste de sanidade teve duração de sete dias, após esse período realizou-se a avaliação dos microrganismos presentes nas sementes com o auxílio de estéreomicroscópio, quando não foi possível a identificação, lâminas com estruturas fúngicas foram preparadas e analisadas com auxilio microscópio ótico. Indica-se a concentração de $50 \%$ do extrato, por proporcionar melhor germinação e IVG as sementes da espécie L. sericeus e reduzir a população de alguns agentes associados às sementes.
\end{abstract}

Palavras-chave: Aspergilus niger, deterioração, armazenamento.

\section{Health and germination treatments of Lonchocarpus sericeus (Poir.) Kunth ex DC}

\begin{abstract}
This research aimed to verify the effects of Gingiber officinale extract on the germination and sanity of seeds of Lonchocarpus sericeus and to encourage the use of natural alternatives in obtaining control of microorganisms associated to the seeds. In order to perform the germination test, the seeds were submitted to the process of overcoming tegumentary dormancy through mechanical scarification with sandpaper number 80, and subsequently submitted to treatments with ginger extract in the following concentrations: $\mathrm{T} 1$ (Control) $=40 \mathrm{ml}$ of sterile $\mathrm{H}_{2} \mathrm{O}, \mathrm{T} 2=10 \mathrm{ml}$ of extract $+30 \mathrm{ml}$ of sterile $\mathrm{H}_{2} \mathrm{O}$, T3 $=20 \mathrm{ml}$ of extract $+20 \mathrm{ml}$ of sterile $\mathrm{H}_{2} \mathrm{O}$, T4 $=30 \mathrm{ml}$ of extract $+10 \mathrm{ml}$ of sterile $\mathrm{H}_{2} \mathrm{O}$ and $\mathrm{T} 5=40 \mathrm{ml}$ of extract. The sanity test lasted seven days, after which the microorganisms present in the seeds were evaluated with the aid of stereomicroscope, when identification was not possible, slides with fungal structures were prepared and analyzed with the aid of an optical microscope. It is indicated the concentration of $50 \%$ of the extract, as it provides better germination and IVG the seeds of the species Lonchocarpus sericeus and reduce the population of some agents associated to the seeds.
\end{abstract}

Keywords: Aspergilus niger, deterioration, storage.

\section{INTRODUÇÃO}

Lonchocarpus sericeus, é espécie conhecida popularmente como ingazeira, falso ingá, entre outros, pertence à família Fabaceae, pode atingir cerca de $20 \mathrm{~m}$ de altura, possui madeira de boa resistência mecânica, exibe baixa frequência em sua faixa de distribuição, e apresenta-se distribuída no Nordeste, Leste do país, Pantanal Matogrossense e Goiás, é árvore característica de matas ciliares e matas costeiras em formações primárias ou secundarias, com produção de frutos de julho a agosto (LORENZI, 2002), e com sementes que apresentam dormência tegumentar (ALMEIDA et al., 2018).

As alterações no código florestal podem possibilitar o aumento na necessidade de produção de mudas para fins de programas de restauração e recuperação ambiental. No entanto para que esses programas possam vir a ter sucesso é necessário, que as mudas a serem instaladas nas áreas a serem reflorestadas apresentem boas qualidades morfológica, fisiológica e sanitária. De acordo com as instruções para análise de sementes, tais atributos podem ser adquiridos quando se trabalha com sementes de boa qualidade (BRASIL, 2009).

A semente é um material genético de alta variabilidade, resultante do cruzamento entre dois gametas (masculino e feminino) Cocucci e Mariath, (2004) e que se constitui uma importante fonte de propagação de espécies florestais e agrícolas. A qualidade desse material pode ser influenciada por fatores, como colheita, beneficiamento, Matias et al. (2014) e armazenamento adequado, Vicente et al. (2016), tais fatores quando não considerados podem influenciar na susceptibilidade das sementes ao

\footnotetext{
Recebido em 05/07/2019, Aceito para publicação em 15/10/2019

${ }^{1}$ Universidade Federal de Campina Grande (UFCG)

*e-mail: lenita-g@live.com
} 
ataque de agentes patológicos, provocar efeitos na longevidade das sementes, e até mesmo deterioração do material propagativo.

As sementes estão susceptíveis a ataques em diferentes fases da sua vida, Oliveira (2012) destaca que as sementes podem ser atacadas em duas etapas: Pré-emergência quando o ataque é feito antes da germinação e pós-emergência das plântulas, tais eventos podem resultar em prejuízos a suas funções vitais e diminuição na qualidade das próprias sementes. A ação de diversos microorganismos, interferem na qualidade sanitária das mesmas e ocasiona danos como: morte em pré-emergência, podridão radicular, tombamento de mudas em condições de viveiro, entre outros (FANTINEL et al., 2017).

Pesquisas relacionadas à sanidade de sementes se tornam pertinente por possibilitarem que sejam conhecidos quais os microrganismos estão presentes no material propagativo, possibilitar que seja indicados tratamentos para o controle adequado dos fitopatógenos e assim, evitar danos futuros com a produção de mudas de baixa qualidade (SOUZA et al., 2013).

Alguns estudos exibem resultados para os principais fungos que causam perda de viabilidade de sementes como os fungos do gênero Aspergillus, Penicillium Rhizoctonia spp, Rhizopus spp, Alternaria spp, encontrados nos resultados de Piveta et al. (2014), outros pesquisas mostraram resultados para espécies nativas da Caatinga como os estudos de Nascimento et al. (2018) e encontram A. niger, A. flavus, A. candidus, e Penicillinum spp, em maior índice de ocorrência. Levando em consideração a demanda futura por mudas de espécies florestais para fins de restauração e recuperação florestal se faz necessário o desenvolvimento de pesquisas que levem em consideração a utilização de produtos de ordem secundária, como os extratos vegetais no controle de fungos, tais pesquisas se apresentam como um viés relevante uma vez que fornecem ao produtor oportunidades de utilizarem produtos de baixo custo, menos onerosos e que causam menos impactos ao meio ambiente.

Diante disto, essa pesquisa teve como objetivo verificar os efeitos do extrato de Gingiber officinale na germinação e sanidade de sementes de $L$. sericeus na redução e/ou eliminação de microorganismos presentes em sementes. Assim como incentivar o uso de alternativas naturais na obtenção de controle de microorganismos.

\section{MATERIAL E MÉTODOS}

O experimento foi realizado no Laboratório de Patologia Florestal, do Curso de Engenharia Florestal, da Universidade Federal de Campina Grande - Campus de Patos-PB. Foram utilizadas sementes de Lonchocarpus sericeus, coletadas em agosto de 2018 no distrito de Iara-Ceará.

Para realização do teste de germinação as sementes da espécie acima citada foram submetidas ao processo de superação de dormência tegumentar através de escarificação mecânica com lixa número 80 , e posteriormente submetidas aos tratamentos com extrato de gengibre nas seguintes concentrações $\mathrm{T} 1($ Controle $)=40 \mathrm{ml} \mathrm{de} \mathrm{H}_{2} \mathrm{O}$ de estéril, $\mathrm{T} 2=10 \mathrm{ml}$ de extrato $+30 \mathrm{ml}$ de $\mathrm{H}_{2} \mathrm{O}$ de estéril, $\mathrm{T} 3=20 \mathrm{ml}$ de extrato $+20 \mathrm{ml}$ de $\mathrm{H}_{2} \mathrm{O}$ de estéril, $\mathrm{T} 4=30 \mathrm{ml}$ de extrato $+10 \mathrm{ml}$ de $\mathrm{H}_{2} \mathrm{O}$ de estéril e $\mathrm{T} 5=40 \mathrm{ml}$ de extrato. Para a determinação das concentrações a serem utilizadas nos referidos tratamentos quantificou-se o volume de água estéril necessário para cobrir uma amostra de 100 sementes de $L$. sericeus (T1) e por proporção os outros tratamentos foram determinados. O extrato hidroalcoólico de Gingiber officinale foi obtido de acordo com a metodologia de (HENRIQUE, 2018).

As sementes foram postas para germinar em recipientes plásticos de cor branca opaca e com dimensões $15 \mathrm{~cm} \times 10 \mathrm{~cm}$ x $4,5 \mathrm{~cm}$, esterilizados com álcool 70, contendo como substrato areia previamente autoclavada e umedecida com água destilada. $\mathrm{O}$ teste de germinação foi conduzido em temperatura ambiente e foi encerrado no $17^{\circ}$ dia, quando se observou a estabilização do processo germinativo. No final do experimento foram calculados a porcentagem de sementes germinadas, anormais, o Î́ndice de Velocidade de Germinação (IVG) e comprimento de parte área e raiz com auxilio régua graduada.

$\mathrm{O}$ teste de sanidade foi realizado a partir do método de papel-filtro "Blotter Test". Foram utilizadas 500 sementes para a instalação do experimento as quais passaram por uma pré-seleção, com objetivo de selecionar as sementes livres de danos visíveis ao olho nu. Foram aplicados os mesmos tratamentos utilizados no teste

As sementes de $L$. sericeus foram imersas nas devidas concentrações por 30 segundos, em seguida levadas para capela de isolamento, previamente desinfectada, sendo colocadas em placas de Petri previamente esterilizadas. Em cada placa foram dispostas 3 folhas de papel-filtro esterilizadas e umedecidas com água estéril. Ao término do processo as placas foram encaminhadas para a câmara de incubação de fungos a temperatura ambiente.

O teste de sanidade teve duração de sete dias, após esse período realizou-se a avaliação dos microrganismos associados nas sementes com o auxílio de estereomicroscópio, quando não foi possível a identificação, lâminas com estruturas fúngicas foram preparadas e analisadas com auxilio 
microscópio ótico, conforme descrição de Barnett e Hunter (1999).

O delineamento estatístico utilizado foi o inteiramente casualizado (DIC) com 5 tratamentos, 4 repetições e 25 sementes em cada repetição, onde os resultados em percentagem foram transformados em arc sen $\sqrt{ }^{-} \mathrm{x} / 100$ e as médias foram comparadas pelo teste de Tukey a um nível de 5\% de significância com auxílio do programa estatístico SISVAR, (FERREIRA, 2011).

\section{RESULTADOS E DISCUSSÃO}

A análise mostrou diferenças significativas para à variável número de sementes germinadas de $L$. sericeus pelo teste de Tukey $(\mathrm{p} \leq 0,05)$, (Tabela 1$)$.

Tabela 1. Porcentagem média de germinação, índice de velocidade de germinação, e comprimento de raiz e parte aérea de Plântulas de Lonchocarpus sericeus submetidas a diferentes concentrações de extrato de Gingiber officinale.

\begin{tabular}{|c|c|c|c|c|}
\hline Tratamentos & Germinação (\%) & IVG & $\mathrm{CRA}(\mathrm{cm})$ & $\mathrm{CPA}(\mathrm{cm})$ \\
\hline Testemunha & $80 a b$ & $3,22 \mathrm{ab}$ & $120.225 \mathrm{a}$ & $175.025 \mathrm{a}$ \\
\hline $25 \%$ de extrato & $86 a b$ & $3,36 \mathrm{a}$ & $138.65 \mathrm{a}$ & $190.075 \mathrm{a}$ \\
\hline $50 \%$ de extrato & $89 a$ & $3,28 \mathrm{a}$ & $132.6 \mathrm{a}$ & $180.475 \mathrm{a}$ \\
\hline $75 \%$ de extrato & $68 \mathrm{~b}$ & $2,43 \mathrm{~b}$ & $141.75 \mathrm{a}$ & $176.25 \mathrm{a}$ \\
\hline $100 \%$ de extrato & $77 \mathrm{ab}$ & $3,10 \mathrm{ab}$ & $145.375 \mathrm{a}$ & $175.525 \mathrm{a}$ \\
\hline
\end{tabular}

Fontes: os autores.

Os melhores resultados para a porcentagem de germinação foram obtidos quando utilizou-se concentrações de $50 \%$ do extrato de gengibre, concentrações acima de $50 \%$ conferiram as sementes da espécie desse estudo germinação inferior a testemunha. Medeiros et al. (2013), estudou o potencial dos extratos de melão-de-sãocaetano (Momordica charantia) e alamanda (Allamanda blanchetti) sobre a micoflora e a qualidade fisiológica de sementes de Pterogyne nitens, e verificou que o uso dos extratos vegetais foi capaz de elevar a germinação da espécie a $71 \%$.

Resultados diferentes dos dessa pesquisa foram encontrados por Cleiton José et al. (2016), onde as altas concentrações do extrato pirolenhoso conferiram efeito inibidor sobre a germinação de Eugenia dysenterica, e Handroanthus serratifolius duas espécies típicas do Cerrado Brasileiro. As concentrações de 25 e $50 \%$ do extrato de gengibre proporcionaram maior índice velocidade de germinação (IVG) em relação a testemunha, e concentrações acima de $50 \%$ do extrato de gengibre reduziram o índice de velocidade de germinação.

Pacheco et al. (2018), estudou o potencial do extrato de Pityrocarpa moniliformis (Benth.) Luckow \& R. W. Jobson (Fabaceae) sob a germinação de Mimosa caesalpiniifolia Benth. (Fabaceae), e também encontrou resultados semelhantes para a variável índice de velocidade de germinação, onde o aumento das concentrações do extrato de Pityrocarpa moniliformis (Benth.) Luckow \& R. W. Jobson (Fabaceae) reduziu a velocidade de germinação da espécie. O uso de extratos vegetais na germinação e sanidade de sementes de espécies florestais e agrícolas vem se intensificando cada vez mais em virtude de ser um método simples e menos oneroso e que não causam prejuízos ao meio ambiente, no entanto estudos com espécies florestais ainda são incipientes principalmente para a espécie $L$. sericeus. Os resultados obtidos aqui reforçam ainda mais a importância de pesquisas que visem os produtos naturais em substituição aos químicos uma vez que esses produtos podem ser tóxicos para determinados de microorganismos, contribuir para a extinção da microflora indesejável e melhorar a qualidade silvicultural das mudas.

A morfologia das plântulas de L. sericeus apresentaram-se pouco diferenciada quando tratadas com extrato de gengibre, no entanto, as concentrações de 25 e $50 \%$ do extrato proporcionaram incrementos maiores na parte aérea das plântulas de L. sericeus enquanto que, concentrações $75 \%$ e $100 \%$ favorecerem melhor o desenvolvimento do sistema radicular, no entanto as diferentes concentrações do extrato de gengibre utilizadas não influenciaram de forma negativa as características morfológicas da espécie, o que 
favorece e reforça a utilização do extrato de gengibre como método alternativo para o controle de fungos .
A tabela 2 exibe os resultados para a microflora de fungos associados nas sementes de L. serceus submetidas a diferentes concentrações de extrato de gengibre (Gingiber officinal).

Tabela 2. Incidência de fungos em sementes de Lonchocarpus sericeus tratadas com extrato de gengibre (Gingiber officinale).

\begin{tabular}{cccccccc} 
Tratamento & A. niger & A. flavus & A. glaucous & A. candidus & Penicillium & Curvularia & Trichoderma \\
\hline Testemunha & $27,2 \mathrm{a}$ & $13,7 \mathrm{a}$ & $14,3 \mathrm{a}$ & $14,7 \mathrm{ab}$ & $0 \mathrm{a}$ & $3,1 \mathrm{a}$ & $3,1 \mathrm{a}$ \\
$25 \%$ de extrato & $27,4 \mathrm{a}$ & $16,3 \mathrm{a}$ & $9,9 \mathrm{a}$ & $39,7 \mathrm{~b}$ & $1,8 \mathrm{a}$ & $1,8 \mathrm{a}$ & $1,6 \mathrm{a}$ \\
$50 \%$ de extrato & $30,3 \mathrm{a}$ & $13,1 \mathrm{a}$ & $40,8 \mathrm{a}$ & $8,1 \mathrm{a}$ & $0 \mathrm{a}$ & $0 \mathrm{a}$ & $0 \mathrm{a}$ \\
$75 \%$ de extrato & $50,2 \mathrm{a}$ & $13,3 \mathrm{a}$ & $19,5 \mathrm{a}$ & $16,9 \mathrm{ab}$ & $0 \mathrm{a}$ & $0 \mathrm{a}$ & $0 \mathrm{a}$ \\
$100 \%$ de extrato & $28,1 \mathrm{a}$ & $35,9 \mathrm{a}$ & $10 \mathrm{a}$ & $21,4 \mathrm{ab}$ & $0 \mathrm{a}$ & $0 \mathrm{a}$ & $0 \mathrm{a}$ \\
\hline
\end{tabular}

\begin{tabular}{cccccccc}
\hline Tratamento & Ovularia & Ovulaniops & Asteromyces & Stigmina & Pithomyse sp & Pleurostronella & Gilmaniella \\
\hline Testemunha & $12 \mathrm{~b}$ & $1,6 \mathrm{a}$ & $8,3 \mathrm{a}$ & $1,9 \mathrm{a}$ & $0 \mathrm{a}$ & $0 \mathrm{a}$ & $0 \mathrm{a}$ \\
$25 \%$ de extrato & $0 \mathrm{a}$ & $0 \mathrm{a}$ & $0 \mathrm{a}$ & $1,6 \mathrm{a}$ & $0 \mathrm{a}$ & $0 \mathrm{a}$ & $0 \mathrm{a}$ \\
$50 \%$ de extrato & $2,5 \mathrm{ab}$ & $2,5 \mathrm{a}$ & $0 \mathrm{a}$ & $0 \mathrm{a}$ & $2,8 \mathrm{a}$ & $0 \mathrm{a}$ & $0 \mathrm{a}$ \\
$75 \%$ de extrato & $0 \mathrm{a}$ & $0 \mathrm{a}$ & $0 \mathrm{a}$ & $0 \mathrm{a}$ & $0 \mathrm{a}$ & $0 \mathrm{a}$ & $0 \mathrm{a}$ \\
$100 \%$ de extrato & $0 \mathrm{a}$ & $0 \mathrm{a}$ & $0 \mathrm{a}$ & $0 \mathrm{a}$ & $0 \mathrm{a}$ & $2,3 \mathrm{a}$ & $2,3 \mathrm{a}$ \\
\hline
\end{tabular}

A microflora encontrada nas sementes de $L$. serceus composta por fungos dos gêneros Aspergilus niger, A. flavus, A. glaucous, A. candidus, que se apresentaram em maiores percentuais, nas diferentes concentrações utilizadas nesse experimento e por fungos dos gêneros Penicilluim, Curvularia, Trichoderma, Ovularia, Ovolanips, Asteromyces, Stigmina Pithtomyse, Pleurostronella, Gimaniella que se apresentaram em menores incidências.

Os fungos dos gêneros A. niger, A. flavus, também foram encontrados em maior ocorrência em sementes de mutamba (Guazuma ulmifolia Lam.), no trabalho de Salles et al. (2018), e também no trabalho de Nascimento et al. (2017), onde esses pesquisadores encontraram microflora semelhante a encontrada nessa pesquisa com maior ocorrência para os gêneros A. niger, A. flavus, A. candidus trabalhando com sementes de espécies nativas da Caatinga, e outros gêneros com menor ocorrência, também semelhantes aos resultados dessa pesquisa. Resultados diferentes desses foram encontrados por Bressan et al. (2018), onde os fungos do gênero Aspergilus foram encontrados em menor frequência. Os fungos do gênero Aspergilus são comumente encontrados em sementes florestais e estão associados às condições de armazenamento das sementes, podendo provocar prejuízos a mesmas como, por exemplo, a deterioração (OLIVEIRA, 2012).

Nenhuma das concentrações das concentrações do extrato de Gengibre (Gingiber officinale), foi capaz de reduzir a incidência dos fungos A. niger, $A$. flavus, A. glaucous, A. candidus, para Sousa et al. (2012), a eliminação dos fitopatogênos nas sementes pode depender de diferentes fatores como por exemplo, localização dos mesmos nas sementes, concentração das substancias utilizadas e o tempo de imersão das sementes nas referidas substancias. As concentrações de $25 \%$ do extrato foram capazes de reduzir a população de Asteromyces, concentrações de $50 \%$ reduziram a população de Stigmina, e concentrações de $75 \%$ do extrato reduziram a incidência de Ovularia, Ovolanips e Pithtomyse.

Para Machado (2000), a erradicação total de alguns grupos pode ser dificultada quando os agentes fitopatogênicos encontram em forma de micélio e infiltrados já nas sementes, ainda de acordo com o mesmo autor um grupo de patógenos pode se manifestar nas formas de contaminação (quando estão presentes em estruturas externas e sem atividade) ou infecção (quando estão infiltrados nas estruturas internas das sementes). Dessa forma, acredita-se que os patógenos os quais o extrato de gengibre não reduziu a incidência já se encontravam nos tecidos internos das sementes parasitando-as.

Algumas estruturas encontradas em diversas sementes não podem ser identificado a olho nu, ou com uso de microscópio estereoscópico, o que dificulta a o processo de análise em sementes florestais, dessa forma, investir em medidas preventivas como a coleta de sementes em matrizes que aparentam boa qualidade genética, realizar o 
beneficiamento e armazenamento de forma adequada, não erradicam os problemas com ataques de fungos em sementes mas podem contribuir para atenua-los.

\section{CONCLUSÃO}

O extrato de Gengibre (Gingiber officinale) pode ser usado como método alternativo para sanidade em sementes de Lonchocarpus sericeus, por não apresentar efeito tóxico na germinação e no desenvolvimento da espécie.

Indica-se a concentração de $50 \%$ do extrato, por proporcionar melhor germinação e IVG às sementes da espécie Lonchocarpus sericeus e reduzir a população de alguns agentes fitopatogênicos. Sugerem-se estudos posteriores com tempo de imersão superiores aos dessa pesquisa.

\section{REFERÊNCIAS}

ALMEIDA, E. P., CUNHA, M. C. L, FERNANDES, S.P.S., NASCIMENTO, A. K. A. Métodos para superação de dormência tegumentar em sementes de Lonchocarpus sericeus (Poir.) Kunt ex dc. (Fabaceae). Tópicos em Engenharia Florestal. Belo Horizonte: Poisson, 2018, p.56-61.

BARNETT, H. L., HUNTER, B. B. Illustred genera of imperfect fungi. 3rd ed. Minnesota: Burgess Publishing Company, 1999. 241 p.

BRASIL. Ministério da Agricultura, Pecuária e Abastecimento. Regra para análise de sementes. Brasília, DF, 2009. 399 p.

BRESSAN, D. F., OLIGINI, K. F., CECHIN, F. E., FUNGHETTO, D. J. Patologia e germinação de sementes de angico-vermelho (Parapiptadenia rígida (benth) brenan) e potencial de óleos essenciais no controle de rhizoctonia sp. In vitro e no tratamento de sementes. Revista Técnico-Científica, Curitiba, n. 10, 2018.

CLEITON JOSÉ, A., ANDRADE, R. J.; PEREIRA, W. V. S., SILVA, N. C. N. FARIA, J. M. R. Efeito do extrato pirolenhoso sobre a germinação de espécies do Cerrado brasileiro. Caderno de Ciências Agrárias, Montes Claros, v. 8, n. 1, p. 62-69, 2016.

COCUCCI, A, E., MARIATH, J. E. A. Gametogênese, fecundação, seleção do gametófito mais apto, embriogênese e diásporo maduro. In: FERREIRA, A.G., BORGHETTI, F. (Orgs.). Germinação: Do básico ao aplicado. Porto Alegre: Artmed, 2004, p.16-28.

FANTINEL, V. S., OLIVEIRA, L. M., CASA, R. T., ROCHA, E. C., SCHNEIDER, P. F., POZZAN, M., LIESCH, P. P., ROMELL, A. R. Fungos associados às sementes de Acca sellowiana: efeitos na qualidade fisiológica das sementes e transmissão. Agrarian, Dourados, v. 10, n. 38, p. 328-335, 2017.
FERREIRA, D. F. Sisvar: versão 5.6. Lavras: UFLA, 2011.

HENRIQUE, G. S. Avaliação do extrato de cravo-daíndia na germinação e na sanidade de sementes de jurema preta e faveleira. 2018. 37f. Trabalho de conclusão de curso (Graduação em Engenharia Florestal) - Universidade Federal de Campina Grande, Paraíba.

LORENZI, H. Árvores brasileiras: manual de identificação e cultivos de plantas arbóreas nativas do Brasil, v.1. Nova Odessa: Instituto Plantarum, 2002. $384 \mathrm{p}$.

MACHADO, J. C. Tratamento de sementes no controle de doenças. Lavras: LAPS/UFLA/FAEPE, 2000.138p.

MATIAS, J. R., OLIVEIRA, G. M.; DANTAS, B. F. Colheita e beneficiamento de algumas espécies da caatinga. Informativo ABRATE, v. 24, n. 3, p. 23-27, 2014

MEDEIROS, J. G. F., NETO, A. C. A., MEDEIROS, D. S., NASCIMENTO, L. C., ALVES, E. U. Extratos Vegetais no Controle de Patógenos em Sementes de Pterogyne nitens Tul. Floresta e Ambiente, Rio de Janeiro, v. 20, n. 3, p. 384-390, 2013.

NASCIMENTO, M. G. R. LOPES, K. P., CÉZAR, M. A.; COSTA, M. M. L., CARDOSO, T. A. L., SOARES, M. G. O. Isolamento de fungos fitopatogênicos em sementes da árvore Caatinga. Revista de la Facultad de Agronomía, La Plata, Argentina, v. 116, n. 2, p. 241 248, 2018.

OLIVEIRA, O. S. Tecnologia de sementes florestais: Espécies Nativas, ed 1. Curitiba, Editora da UFPR, 2012. 404 .

PACHECO, M. V., Felix, F. C., Medeiros, J. A. D., Nunes, S. L., Castro, M. L. L., Lopes, A. L. S., Souza, W. M. A. T. Potencial alelopático dos extratos de folhas e frutos de Pityrocarpa moniliformis sobre a germinação de sementes de Mimosa caesalpiniifolia. Revista Agroecossistemas, Pará, v. 9, n. 2, p. 250-262, 2018.

PIVETA, G., MUNIZ, M. F. B., REINIGER, L. R. S., DUTRA, C. B., PACHECO, C. Qualidade sanitária e fisiológica de sementes de aroeira-preta (Lithraeamolleoides) submetidas a métodos de superação de dormência Ciência Florestal, Santa Maria, RS, v. 24, n. 2, p. 289-297, 2014.

SALES, N. L. P., COTA, C.G., FREITAS, F. G. R., MOREIRA, J. L., CARVALHO, L. R., MOREIRA, C. D. D.; BARROSO, P. D. Germinação, sanidade e tratamento de sementes de Guazuma ulmifolia Lam./Germination, sanity and seed treatment of Guazuma ulmifolia Lam. Caderno de Ciências Agrárias, Montes Claros, v. 10, n. 2, p. 46-52, 2018.

SOUZA, L. M. S., SIlVA, J. B., GOMES, N. S. B. Qualidade sanitária e germinação de sementes de copaíba. 
Bioscience Journal, Uberlândia v.29, n.5, p. 1524-1531, 2013.

VICENTE, D., OLIVEIRA, L. M., TONETTI, O. A. O., SILVA, A. A., LIESCH, P. P., ENGEL, M. L. Viabilidade de sementes de Ocotea puberula (Rich.) Ness ao longo do armazenamento. Floresta e Ambiente, Rio de Janeiro, v. 23, p. 418-426, 2016. 